\title{
Comparison of cryogenic low-pass filters
}

\author{
M. Thalmann, ${ }^{1}$ H.-F. Pernau, ${ }^{1, \text { a) }}$ C. Strunk, ${ }^{2}$ E. Scheer, ${ }^{1}$ and T. Pietsch ${ }^{1,3, b)}$ \\ ${ }^{1}$ Department of Physics, University of Konstanz, 78464 Konstanz, Germany \\ ${ }^{2}$ Institute of Experimental Physics, University of Regensburg, 930253 Regensburg, Germany \\ ${ }^{3}$ Zukunftskolleg, University of Konstanz, 78464 Konstanz, Germany
}

(Received 10 July 2017; accepted 19 October 2017; published online 17 November 2017)

\begin{abstract}
Low-temperature electronic transport measurements with high energy resolution require both effective low-pass filtering of high-frequency input noise and an optimized thermalization of the electronic system of the experiment. In recent years, elaborate filter designs have been developed for cryogenic low-level measurements, driven by the growing interest in fundamental quantum-physical phenomena at energy scales corresponding to temperatures in the few millikelvin regime. However, a single filter concept is often insufficient to thermalize the electronic system to the cryogenic bath and eliminate spurious high frequency noise. Moreover, the available concepts often provide inadequate filtering to operate at temperatures below $10 \mathrm{mK}$, which are routinely available now in dilution cryogenic systems. Herein we provide a comprehensive analysis of commonly used filter types, introduce a novel compact filter type based on ferrite compounds optimized for the frequency range above $20 \mathrm{GHz}$, and develop an improved filtering scheme providing adaptable broad-band low-pass characteristic for cryogenic low-level and quantum measurement applications at temperatures down to few millikelvin. Published by AIP Publishing. https://doi.org/10.1063/1.4995076
\end{abstract}

\section{INTRODUCTION}

The development of mesoscopic quantum electronic devices requires ultra-sensitive measurements of their transport characteristics, performed at cryogenic conditions due to the low relevant energy scales. Examples include single electron tunneling devices, ${ }^{1-3}$ quantum electrodynamics in superconducting qubits and Josephson junctions, ${ }^{4-6}$ as well as single atomic- and molecular contacts ${ }^{7-9}$ and nuclear spin systems ${ }^{10-15}$ among many other experiments mainly performed at temperatures well below $1 \mathrm{~K}$. At these temperatures, the suppression of the electronic temperature in the system via thermal anchoring of the leads and their filtering to isolate the sample from the room-temperature electromagnetic environment has always been a difficult task, especially for complex measurement applications involving large sets of mixed low- and high-frequency signal lines. Therefore, in this report, we collect the experience of several years of filter development in our lab with respect to the design and technology of low-level electronic measurement circuits and their thermal anchoring for quantum transport experiments in the few millikelvin regime and in magnetic fields up to about $10 \mathrm{~T}$. In conventional, wet cryogenic systems space constraints have to be considered, which affect the design of efficient filters and thermal anchors. Driven by recent developments in "dry" dilution cryostats, ${ }^{16}$ transport experiments in solids at base temperatures of $10 \mathrm{mK}$ and even below have become a standard technique. However, the electronic temperature of the device under test is often considerably larger since adequate filtering of spurious high frequency (HF) signals and thermalization to the cryogenic bath are not provided. Below $1 \mathrm{~K}$,

\footnotetext{
a)Present address: Fraunhofer Institute for Physical Measurement Techniques (IPM), 79110 Freiburg, Germany.

b)Electronic mail: torsten.pietsch@uni-konstanz.de
}

most of the thermal transport in solid matter is carried by the electronic system $\left(\propto T^{2}\right)$ rather than phonons $\left(\propto T^{5}\right)$ or radiative heat transfer. ${ }^{17,18}$ Hence, thermalizing the sample's electron gas to the bath at temperatures below $100 \mathrm{mK}$ is mainly via the electrical wires and can be a challenging task. Moreover, shielding the experiment from room temperature electromagnetic radiation requires heavy electronic low-pass filtering at various temperature stages. In a first approximation, the electronic noise at a given maximum frequency $v$ can be translated into the effective temperature of the electronic system via $h v=k_{B} T$ and hence $v=20 \mathrm{GHz}$ corresponds to a temperature of $T \approx 1 \mathrm{~K}$. Glattli et al. ${ }^{19}$ calculated the required electrical damping $A(v)$ to shield the "cold" electronic system at $T_{c}$ from the hot source at $T$ and its corresponding "hot" frequency $v$ according to the following equation:

$$
A(v)=\frac{e^{\frac{h v}{k_{B} T}}-1}{e^{\frac{h v}{k_{B} T_{C}}}-1} .
$$

Hence, shielding an experiment at $T_{c}=250 \mathrm{mK}$ from the thermal radiation produced at $4 \mathrm{~K}$ requires only a damping rate of about $-44 \mathrm{~dB}$ at $20 \mathrm{GHz}$. However, shielding $T_{c}=25 \mathrm{mK}$ from the radiation of $T=4 \mathrm{~K}$ requires a minimal attenuation of $A(v)=-50 \mathrm{~dB}$ at frequencies above $521 \mathrm{MHz}$ and a damping of $A(v)=-345 \mathrm{~dB}$ at $v=20 \mathrm{GHz}$ (see Fig. 1).

Such high attenuation factors are difficult to achieve using a single filter stage and without impairing the measurement signal. Therefore, often a cascade of filters is used consisting of at least three filter stages, typically installed at $4 \mathrm{~K}$, at $1 \mathrm{~K}$, and at the base temperature of the cryostat. Also, a good thermal anchoring of the wires at the filter stages, which should be well thermalized at their respective bath temperature, is mandatory. Herein, we introduce an overall filtering concept and an improved distributed elements 


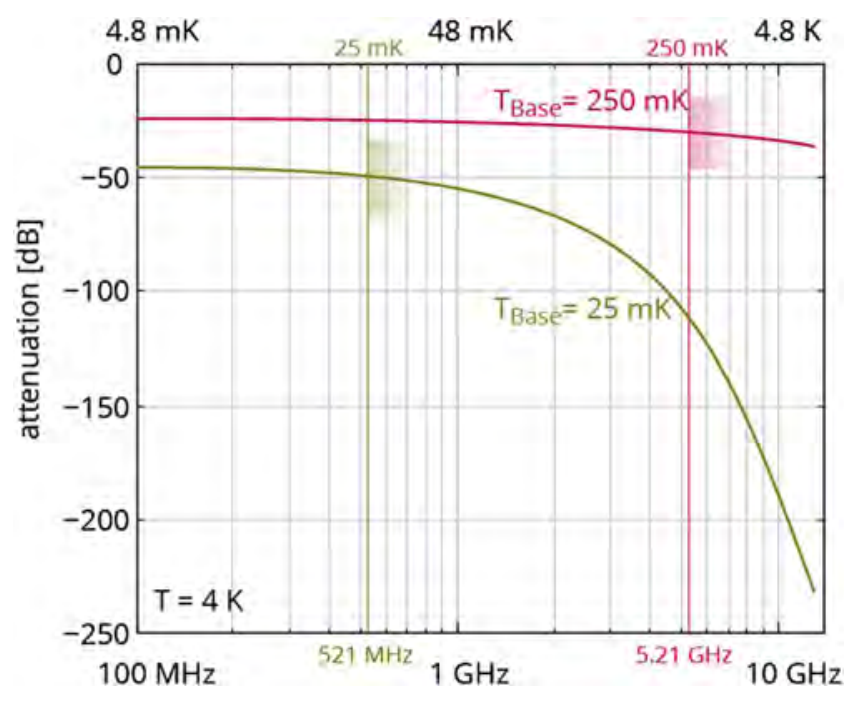

FIG. 1. The attenuation required to shield an experiment at two different base temperatures from a $4 \mathrm{~K}$ reservoir according to Eq. (1).

microstripline filter based on magnetic losses in a ferrite compound. This filter type performs at least as good as commonly used copper powder filters but is more robust, easier to fabricate, and provides tailored filter characteristics with higher attenuation at high frequencies above $10 \mathrm{GHz}$. All filter concepts presented herein can be applied to single coaxial measurement lines as well as to multipole circuits. Wherever practical, we focus our discussion on the popular twisted-pair wiring layout used in DC and low-frequency electronic quantum transport measurements in cryogenic environments.

\section{FILTER TYPES, LOSS COMPONENTS, AND PRINCIPLE CONSIDERATIONS}

Within the past decades, a number of different cryogenic filter types have been developed to attenuate spurious HF components in the signal lines and thermalize the electrical wiring. Popular filter designs include discrete lumped element filters $^{20,21}$ as well as metal powder filters, ${ }^{22-26}$ coaxial- and lossy transmission line filters ${ }^{27-36}$ as well as microfabricatedand distributed element filters ${ }^{37-41}$ among other more exotic variants. An overview over different filter concepts is given in Ref. 20.

The most common low-pass filters are simple resistivecapacitive (RC), resistive-inductive (RL), and resistivecapacitive-inductive (RCL) elements. ${ }^{21}$ The latter two are a special case of low-pass filters of second order, which can be described as a one-pole filter with a cutoff frequency $\omega_{\mathrm{r}}$, if the damping factor fulfils $D=\frac{R}{2} \cdot \sqrt{\frac{C}{L}}>1$; otherwise, i.e., when $D<1$, a resonance occurs at $\omega_{r}=\frac{1}{\sqrt{L C}}$.

Although these filters theoretically follow a one-pole characteristic, in practice, a low attenuation is observed at higher frequencies. Therefore, simple RC or RCL filters are only recommended at frequencies up to several MHz. Signal components above $1 \mathrm{GHz}$ can hardly be handled by this filter type with the notable exception of metal capillary filters discussed in Sec. IV. These capillary filters are essentially capacitive filters composed of a thin coaxial conductor in a capillary tube filled with either conductive or dielectric absorber material. Such micro-coaxial wires with a dielectric thickness of only a few microns yield a high capacitance similar to the commercially available Thermocoax ${ }^{\mathrm{TM}}$ wires well established in cryogenic quantum transport measurements, ${ }^{19,20,35}$ however, without the drawbacks of the brittle and hygroscopic $\mathrm{MgO}$ dielectric employed in Thermocoax wires.

Another common filter type for higher frequency ranges is a metal powder filter, which was developed in the late 1980s by Martinis et al. ${ }^{26}$ The filter consists of an insulated wire with diameter in the order of $50 \mu \mathrm{m}$, which is surrounded by a metal powder, mostly copper, with an optimal grain size of about $30 \mu \mathrm{m}^{22}$ to $64 \mu \mathrm{m} .{ }^{20}$ This filter type is frequently used in low temperature physics and some novel design concepts ${ }^{23-25}$ have been presented in recent years. In powder filters, the low-pass filtering results from regenerated induced vortex currents in the surface of the metal powder grains. Hence, these filters operate on the basis of the skin-effect of HF alternating currents and the large effective surface in the metal powder. Besides their low-pass filtering characteristics, powder filters are state of the art in many cryogenic systems whenever a thermal anchoring of the measurement wiring is concerned. ${ }^{42,43}$ Due to their importance and widespread use, an improved design of metal powder filters is introduced in Sec. III C, and their performance is discussed in Sec. V.

With the development of hybrid microwave integrated circuits (MICs) over the last 40 years, a fundamental understanding of the behavior of electromagnetic waves in integrated microscopic structures and circuits was developed. There are now typical low-pass-structures that are designed using the lumped element model which can be transformed into a distributed element filter, i.e., a transmission line filter, ${ }^{44}$ via Richard's transformation ${ }^{45}$ using Kuroda identities. ${ }^{46,47}$ These filters again work capacitively and most MIC low-pass filter concepts are designed for only a narrow frequency band. However, Vion et al. ${ }^{41}$ showed the possibility to create a wideband low-pass filter using distributed elements on lossless materials, which spurred the design of various types of microfabricated wide-band low-pass filters ${ }^{38,39}$ based on integrated circuits.

An alternative approach to achieve effective broad-band filtering is to use a lossy material in the surrounding of the conducting structure. The main part of the electric and magnetic fields is constrained within the substrate. To reach a high loss in an integrated circuit element, one can either employ a lossy substrate or a very thin substrate within a lossy environment. ${ }^{25}$ The loss of a dielectric material can be described by the ratio of the complex permittivity $\varepsilon$ and permeability $\mu$ through the dielectric loss tangent $\tan \delta_{e}=\frac{\varepsilon^{\prime \prime}}{\varepsilon^{\prime}}$ and magnetic loss tangent $\tan \delta_{m}=\frac{\mu^{\prime \prime}}{\mu^{\prime}}$, respectively, where $\varepsilon^{\prime}, \mu^{\prime}$, and $\varepsilon^{\prime \prime}$, $\mu^{\prime \prime}$ denote the real and the imaginary parts of $\varepsilon$ and $\mu$. The dielectric- and magnetic loss tangents are of course highly material dependent. By taking advantage of this influence of the environmental dielectric material, it is possible to create simple broad-band low-pass transmission line filters ${ }^{29}$ based on a high magnetic loss tangent as demonstrated in Sec. VI. The principle is similar to filters with a metal powder loaded silicon or epoxy compound. However, in this case, a ferrite 
powder is used to produce a compound with a high magnetic loss tangent $\tan \delta_{m}$. Notably, suitable compounds are available commercially and are compared to the custom-made absorber materials. In essence, the concept of magnetically lossy transmission lines is known for many years, ${ }^{31,36}$ but for a long time, their potential in wide-band cryogenic lowpass filtering applications has not been realized. Finally, we show that combining two different filter concepts operating at different frequencies yields a filter with almost ideal cutoff and ultra-wideband low-pass filtering, which results in excellent HF noise rejection and very low electron temperatures. In Sec. III, we first introduce the main filter concepts discussed herein and present their versatile and robust technical realizations. Then, in Secs. IV-VI, the performance of these filters at different frequencies is evaluated and their advantages are discussed.

\section{FILTER DESIGN AND CONSTRUCTION}

\section{A. Metal-powder RC-filters}

In order to achieve good low-pass filtering above a welldefined cutoff frequency in the $\mathrm{kHz}$ to $\mathrm{MHz}$ range, $\mathrm{RC}$ and LC filters are the most commonly used filter types. Usually, since a single RC element has a rather flat cutoff flank, it is recommended to employ higher order filters by combining several RC elements. Common higher-order RC filter designs are called "T-filters" and " $\pi$-filters," which are named according to their shape. In a T-filter, a central capacitance is placed between two resistors, while a $\pi$-filter is composed of two capacitances and three resistances. For example, we employ a 2 nd order $\mathrm{RC}$ filter board in $\pi$-geometry mounted at base temperature below $30 \mathrm{mK}$ in our dilution refrigerator (see Fig. 2), providing a cutoff frequency in the $\mathrm{kHz}$ range in the twisted pair DC measurement lines directly before the sample under test. A dual-channel printed circuit board (PCB) filter is mounted in a solid copper housing filled with copper powder. Here the powder does not provide filtering but ensures good thermalization of the surface-mounted-device (SMD) components and wires. This rugged and compact filter design can be easily adapted to the specific requirements, such as higher order filters and larger number of measurement lines.

The dimension of the SMD elements on the PCB filter board is chosen to yield a cutoff frequency of $150 \mathrm{kHz}$. Typically, in the transmission spectrum above $50 \mathrm{MHz}$, the attenuation decreases continuously. Since the attenuation is already significantly reduced at frequencies of $100 \mathrm{MHz}-1$ $\mathrm{GHz}$, which corresponds to the frequency where copper powder filters become most efficient, an additional RC filter with a higher cutoff frequency in a range of $200 \mathrm{MHz}<v<1 \mathrm{GHz}$ is needed for intermediate frequencies. However, in the following, we will introduce alternative filter concepts that can be combined to achieve sufficient low-pass filtering for quantum transport measurements at temperatures as low as $10 \mathrm{mK}$ across the entire spectrum up to very high frequencies.

\section{B. Single-coaxial and twisted-pair capillary filters}

A capillary tube engineered twisted pair filter can be described as a two channel semi-rigid coaxial cable with a very
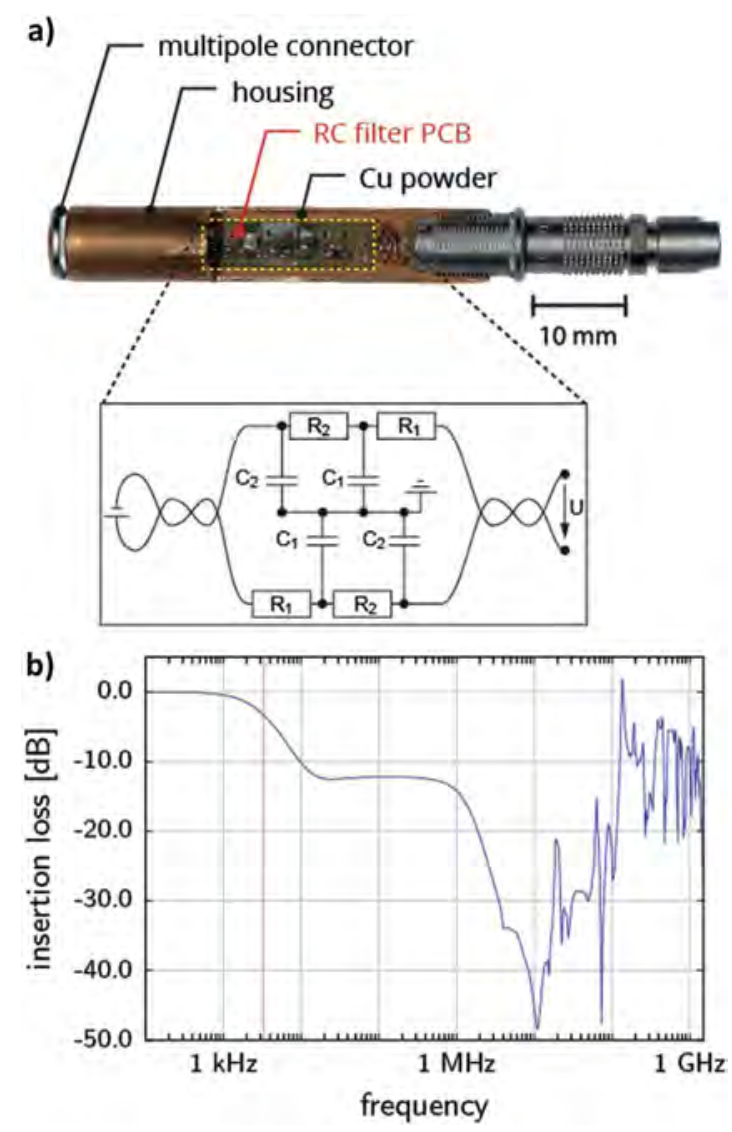

FIG. 2. 2nd order RC filter for bipolar voltage measurement. (a) Photograph of the device and corresponding circuit diagram (b) measured insertion loss spectrum of a filter with $\boldsymbol{R}_{1}=100 \Omega, \boldsymbol{R}_{2}=500 \Omega, \boldsymbol{C}_{1}=33 \mathrm{nF}$, and $\boldsymbol{C}_{2}=33 \mathrm{nF}$ providing a cutoff at $3.0 \mathrm{kHz}$.

thin dielectric sheath. First, commercially available stainlesssteel capillaries with an outer diameter of $300-400 \mu \mathrm{m}$ and a wall thickness of $100 \mu \mathrm{m}$ are cut to length and polished inside mechanically by pulling a $50 \mu \mathrm{m}$ thick steel wire featuring sharp bends through the tube multiple times. After cleaning the capillary tube with isopropanol, a $50 \mu \mathrm{m}$ thick single- or twisted-pair polyimide insulated wire (copper, Manganin ${ }^{\circledR}$, brass or $\mathrm{NbTi}$ ) is soldered end to end to the steel wire and pulled through the capillary, as shown in Fig. 3. Manganin is the trade name of an alloy consisting of $82 \%-84 \%$ copper, $12 \%-15 \%$ manganese, and $2 \%-4 \%$ nickel. It is often used as a cryostat wiring material if high and almost temperature independent lead resistances are required. However, as a consequence of the relatively high resistivity of $\rho=48 \mu \Omega \mathrm{cm}$, Manganin has low thermal conductance. The particular properties of Manganin wires for filter aspects will be discussed in Sec. V.

The space inside the capillary is filled with conductive epoxy by embedding the measurement leads in silver epoxy EccoShield ES 100 GR (ES) from Emerson \& Cuming Microwave Ltd., When feeding the silver-epoxy coated wires into the capillary tube, the adhesive forms a conductive sheath around the wires and firmly bonds to the capillary wall, while the volatile solvent evaporates within few hours of drying at ambient conditions. To remove the solvent more effectively, moderate annealing at elevated temperatures of $80^{\circ} \mathrm{C}$ for $3 \mathrm{~h}$ 

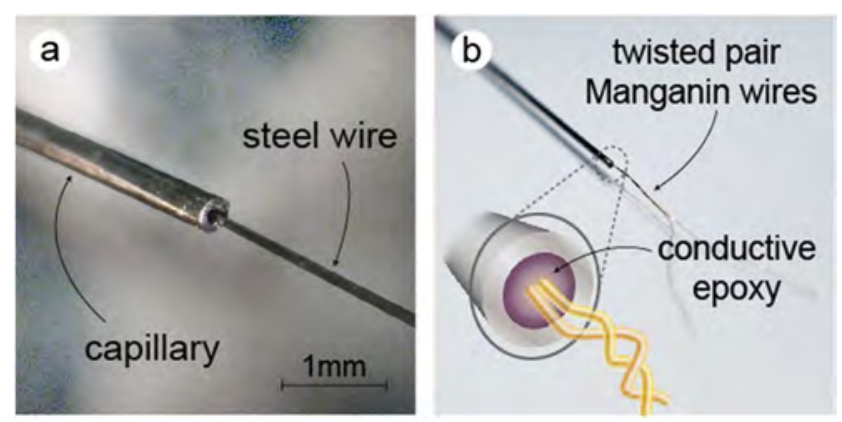

FIG. 3. (a) The capillary and the inserted steel wire. (b) The Manganin wires get pulled through the capillary.

can be used. Before mounting the wire assembly in the cryostat, suitable plugs (coax or multipole connectors) are soldered onto the leads, while the capillary tube forms the guard, which is thermally and electrically anchored in the cryostat.

\section{Copper powder filters}

All metal powder filters basically consist of a long wire in a housing (Fig. 4), which is filled with a metal powder dissipating the evanescent HF components due to the skin effect. The filter characteristics depend on a large number of parameters, such as wire length and coil geometry as well as powder type and grain size. To evaluate the optimal design, we investigated different aspects: (i) the type and granularity of metal powder and (ii) the type of wires used. Two different types of metal powder are tested: stainless steel powder (SSP) and copper powder (CuP) in combination with Manganin or copper transmission wires. A two-channel filter is prepared in the following way. First the two leads are twisted to a pair to reduce the mutual inductance by minimizing the area enclosed by the wires. Then the twisted pair is wound into a coil with special geometry, where the inductance is compensated by the converse direction of the subsequent winding layer [see Fig. 4(d)].

In our design, a coil length of about $15-20 \mathrm{~mm}$ corresponds to a total wire length of $2.5-3 \mathrm{~m}$, when a $50 \mu \mathrm{m}$ thick wire is used. A long wire is important because the filter performance scales favorably with the wire length. The

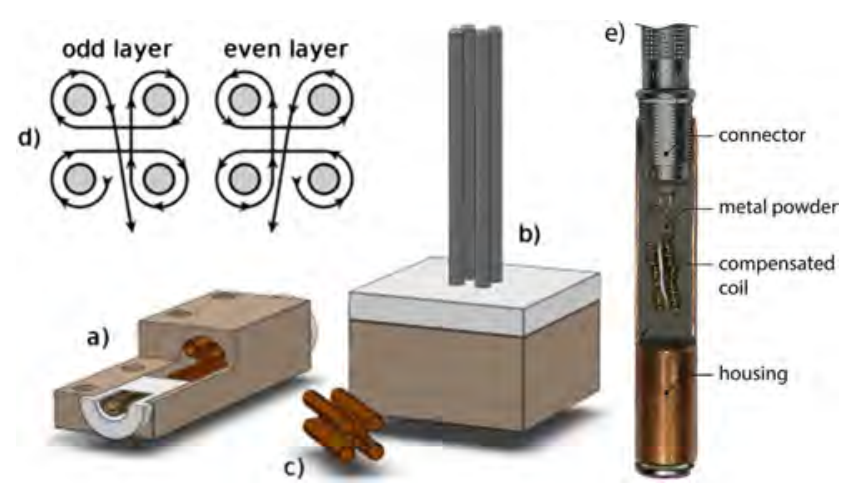

FIG. 4. Schematic assembly of a powder filter. (a) Sketch of the device components, the space between the filter element and housing is filled with metal powder, (b) winding tool with stripping plate, (c) filter coil with induction compensation via opposite directionality of even-odd winding layers (d). (e) Photograph of a length-cut through the final filter device.

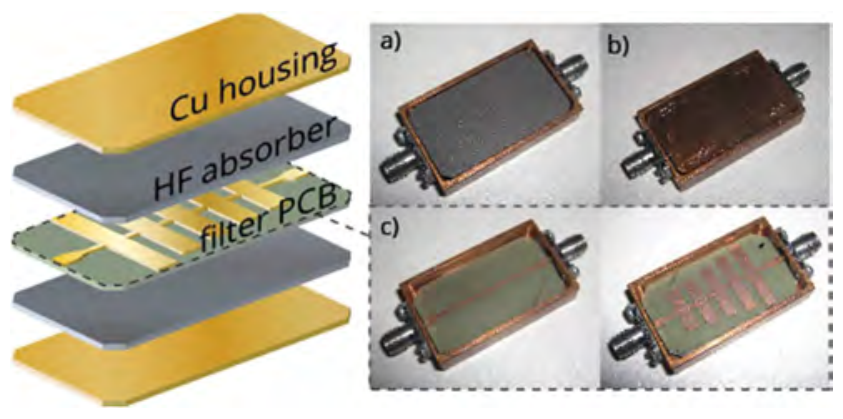

FIG. 5. Principle design of distributed element low-pass filters in a lossy environment. (a) Ferrite compound absorber material (b) copper test fixture, (c) stripline and harped filter elements on a thin FR4 PCB.

compensated coil is mounted in a metal housing, which is filled tightly with metal powder before hermetically sealing the assembly by fitting the electrical connectors on either side. Besides the electronic low-pass filtering, the tight packing of the measurement wire in metal powder also ensures good thermal anchoring of the leads in the cryostat. It is worth noting that the design can be used without much modification to fabricate multipole filters with up to 10 individual measurement leads, which can be advantageous for applications in experiments with severe space constraints. Moreover, the compensated winding scheme, which yields a vanishing net inductance, is a great advantage for measurement applications in magnetic fields.

\section{Ferrite compound filter}

A PCB filter in its simplest form can be just an integrated low-pass element on a circuit board. However, here we use this expression for distributed element circuits with an additional loss component due to a lossy environment-in our case due to a ferrite compound. A simple test fixture made of copper and SMA ${ }^{\circledR}$ connectors on either side is used to evaluate and compare the performance of filter boards with different circuit geometries and different compositions of the absorber compound. Two brackets and a copper lid tightly fix the distributed filter element on a $0.125 \mathrm{~mm}$ thick FR4 PCB sandwiched between two $1 \mathrm{~mm}$ thick pads of absorber material within the housing (Fig. 5). The absorber compounds are custom-made by casting thin mats of silicone rubber incorporating large fractions of ferrite powders. In Sec. VI, we evaluate the HF characteristics of these filters with different absorber compounds compared to commercially available HF absorbers. Then, in Sec. VI, we introduce a modular set of sealed filter elements for cryogenic low-level transport measurements.

\section{PERFORMANCE OF CAPILLARY FILTERS}

As indicated above, the semi-rigid micro-capillary wiring often used in cryogenic environments is not only useful for low-noise electronic measurements but can also serve as a novel type of filter with superior characteristics compared to standard coaxial measurement lines. The loss of this special kind of a coaxial cable essentially depends on its capacitance and therefore the geometry and properties of the dielectric used 
in the construction. Here we show that the use of a conductive HF absorber results in a lower cutoff frequency and a much steeper flank than the standard silicone-filled capillary wires or commercial Thermocoax wires. Because a coaxial cable resembles a real capacitor, with a finite ESL (equivalent series inductance) and ESR (equivalent series resistance), the loss tangent is given by $\tan \delta=R_{E S R} /\left(\omega L_{E S L}-1 / \omega C\right)$.

Due to the application of a conductive HF absorber made of EccoShield by Emerson \& Cuming Ltd., the shield is electrically very close to the wire with a dielectric thickness given by the thickness of the polyimide insulation on the bare wires, which is approximately $7.0 \pm 2.0 \mu \mathrm{m}$ while the wire's diameter is $50 \mu \mathrm{m}$. The dielectric constant of polyimide is approximately $\varepsilon \approx 3.4 \varepsilon_{0}$ around $1 \mathrm{GHz}$ and since polyimide is not magnetic, its permeability is $\mu=\mu_{0}$. Hence at a given surface resistance $R_{s}=\sqrt{\frac{\omega \mu}{2 \sigma}}$, it is possible to estimate the electric characteristics $\mathrm{L}, \mathrm{C}$, and $\mathrm{R}$ using the simplified expressions according to Pozar, ${ }^{48}$

$$
L=\frac{\mu}{2 \pi} \ln \frac{b}{a} \mathrm{H} / \mathrm{m}, \quad C=\frac{2 \pi \varepsilon}{\ln \frac{b}{a}} \mathrm{~F} / \mathrm{m}, \quad R=\frac{R_{s}}{2 \pi}\left(\frac{1}{a}+\frac{1}{b}\right) \Omega \mathrm{m} .
$$

Here $a=25 \mu \mathrm{m}$ is the radius of the bare wire and $b=32$ $\pm 2 \mu \mathrm{m}$ is the radius of the wire including the insulation sheath. Therefore, this capillary should have an inductance $L$ of $49.2 \mathrm{nH} / \mathrm{m}$ and a capacitance $C$ of $788 \mathrm{pF} / \mathrm{m}$. For comparison, we fabricate a set of different capillary wire filters of $1 \mathrm{~m}$ length using different conductor and dielectric materials. Figure 6 shows the transmission characteristic of single-wire capillary filters made of copper and Manganin wires at $77 \mathrm{~K}$ and $300 \mathrm{~K}$. The attenuation is excellent and reaches the noise floor at frequencies of $900 \mathrm{MHz}$ with copper wires and $570 \mathrm{MHz}$ with Manganin wires. In contrast, the measurement of a $\mathrm{Cu}$ coax wire filled with silicone instead of ES shows much weaker attenuation across the entire spectrum, which does not reach the noise floor of our analyzer at all. Moreover, the siliconefilled capillary wires show unpredictable characteristics and a reduced damping at frequencies above $10 \mathrm{GHz}$, whereas the

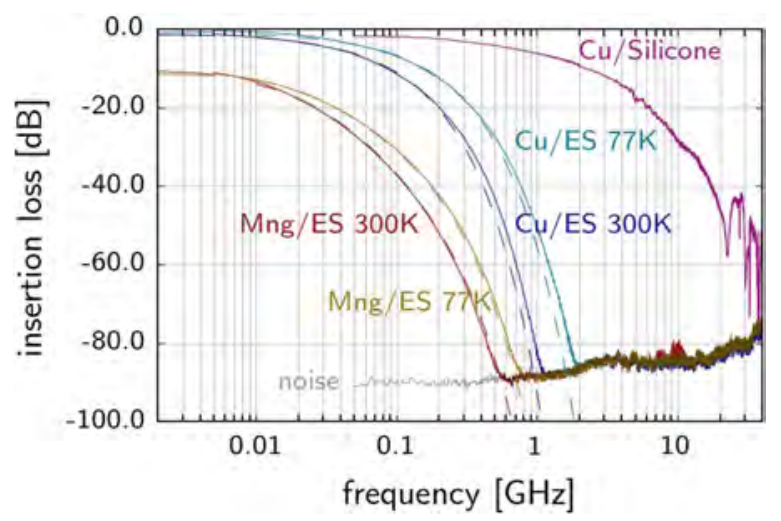

FIG. 6. Comparison of transmission spectra of three different capillary filters of $1 \mathrm{~m}$ length. Simple silicone filled capillaries show a weak attenuation and do not reach noise level at frequencies below $40 \mathrm{GHz}$. In contrast, the insertion loss of capillaries with conductive epoxy reaches the noise level at $900 \mathrm{MHz}$ with copper wires and at $570 \mathrm{MHz}$ with Manganin wires. The dashed lines are fits to the data according to Eq. (2) to extract the loss tangent and the electric characteristics of the filter. attenuation of the ES filled wires remains under the noise floor up to very high frequencies.

A simple RCL-transfer function cannot be used for fitting the spectra because the relevant parameters $\mathrm{R}, \mathrm{C}$, and $\mathrm{L}$ are highly frequency dependent. While a precise theory describing such assemblies exists, Spietz and co-workers ${ }^{49}$ introduced a simple model based on the description of the electrical performance of transmission lines, ${ }^{48}$ which yields an expression for the transfer function,

$$
\left|S_{21}\right|^{2}=\left|\cosh (\gamma l)+\frac{1}{2}\left[\frac{Z_{0}}{Z_{\text {net }}}+\frac{Z_{\text {net }}}{Z_{0}}\right] \cdot \sinh (\gamma l)\right|^{-2} .
$$

Here $l$ is the total length of the assembly, $Z_{\text {net }}$ is the impedance of the transmission line, and $\gamma=\sqrt{(R(\omega)+i \omega L)(G(\omega)+i \omega C)}$ is the frequency dependent propagation constant with the shunt conductance $G=\omega C \tan \delta$, the impedance $L=\epsilon \mu_{0} / C$, and the common mode resistance of the filter $R=R_{\text {in }}+\mathrm{R}_{\text {out }}$, where $\varepsilon=3.4 \varepsilon_{0}$ is the dielectric constant of the polyimide insulation. The measured filter characteristic is fitted using Eq. (2), to extract the loss tangent $\tan \delta$ and the effective capacitance of the assembly. Earlier reports on twisted pair tapeworm filters indicate a loss tangent of 0.01 and a specific capacitance of about $200 \mathrm{pF} / \mathrm{m} .{ }^{49}$ Notably, the $\tan \delta$ of our twisted pair capillary filters is 0.53 for copper wires and 0.48 for Manganin wires at $300 \mathrm{~K}$, with a reduction of $15 \%$ at low temperatures $(77 \mathrm{~K})$ in both cases. Such high loss tangents are achieved by embedding the wire in a conducting medium, which also gives rise to a high capacitance of the assembly. However, compared to our first estimates of $788 \mathrm{pF} / \mathrm{m}$, the effective capacitance of the assembly extracted from Eq. (2) is $145 \mathrm{pF} / \mathrm{m}$ for copper and $172 \mathrm{pF} / \mathrm{m}$ for Manganin wire filters. This strong deviation by a factor of five from the geometrical estimate indicates that the capillary tube is only incompletely filled with conductive epoxy. As a result, the thickness of the dielectric and its dielectric constant (polyimide or vacuum) changes along the capillary wire and the average filling is reflected in the attenuation spectrum. A simple estimate yields a filling fraction of approximately $15 \%-20 \%$ of the capillary tube, which could be improved by optimizing the fabrication method. Especially the evaporation of volatile components of the conductive epoxy results in voids in the filling and perhaps incomplete coverage of the twisted pair wire assembly within the capillary tube. Nevertheless, even this partial filling yields significantly improved filter characteristics compared to silicone-filled coaxial transmission lines. In essence, these capillary wire filters show a very sharp low-pass characteristic due to the high intrinsic capacitance of this type of filter as well as the improved loss tangent compared to earlier reports. The advantages over other filter concepts are that the measurement lines themselves provide the good filtering characteristics, which means that no additional space in the cryostat is consumed by a discrete filter element and the thermal load on the system is significantly reduced. Moreover, the thermal anchoring concept is very straightforward due to the relatively large thermal conductivity of the entire assembly and the thin dielectric sheet. This filter concept can be applied to single coax or twisted pair measurement lines without further modification. However, in some measurement applications, where time resolution is an 
issue, the high lead capacitance may be obstructive. Moreover, the fabrication of long and especially thin $(d<300 \mu \mathrm{m})$ capillary filters filled with conductive adhesive is difficult, due to the risk of breaking the wire's insulation, which will cause a shortcut to cryostat ground.

\section{METAL POWDER FILTERS}

Metal powder filters are commonly used in cryogenic transport measurement applications, where thermal anchoring and low-pass filtering are concerned. Due to their popularity, we investigate different types of powder filters and compare their performance. One major drawback of this type of filters, however, remains as follows: The filter coils are almost always wound by hand and depending on the complexity of the coil geometry there are inhomogeneities in the windings. Since the overall filter performance is very sensitive to the winding and deformation of the coil, the exact filter characteristics are hard to predict or reproduce. One important aspect is that the inductance of the coil grows proportional with frequency below resonance frequency $v_{r}=\frac{1}{2 \pi \sqrt{L C_{p}}}$. The parameter $C_{p}$ is the self-capacitance and $L$ is the inductance of the coil. Therefore the best results can be obtained by keeping the self-capacitance as low as possible, which is the reason for employing the complex geometry shown in Fig. 4(d) rather than a cylindrical coil sometimes found in the literature. ${ }^{23,24}$ A low self-capacitance can also be achieved by increasing the distances between subsequent winding layers, reducing the dielectric constant of the material between windings, a low permittivity of winding reel, or a larger separation between windings and winding reel. In order to optimize the filter design, we studied in detail the following aspects of powder filters, while keeping the design and coil geometry nominally the same.

\section{A. Wire dimensions}

The difference of the surface area of wires with radii differing by a few tens of $\mu \mathrm{m}$ is almost negligible and hence their attenuation behavior is very similar. Furthermore, a thicker wire does not have a higher AC conductivity at high frequencies because the resistance is largely determined by a thin surface sheet due to the skin effect. Hence the AC resistance changes only, if the wire radius is in the range of the skin-depth value. However, in most cases, this regime is hard to reach. For example, a Manganin wire has a skin depth $\delta \approx \sqrt{\frac{2 \rho}{\mu_{0} \omega}}$ (with $\rho$ the resistivity) of only $25 \mu \mathrm{m}$ at $174 \mathrm{MHz}$. It has been established that a shorter wire leads to a flatter flank in the attenuation behavior. Moreover, a thicker wire also translates into larger coils at a given wire length and hence a thinner wire favors a compact filter design. In the optimal case, the thickness should be slightly larger than the skin depth at the desired cutoff frequency.

\section{B. Wire material}

Two different wire materials are investigated: copper and Manganin. Both wires have a diameter of $50 \mu \mathrm{m}$ and a nominal polyimide insulation sheath of 6-10 $\mu \mathrm{m}$. Due to the higher resistivity of Manganin, its damping factor is larger and thus the attenuation flank is steeper, while copper wires show a flatter cutoff [see Fig. 8(c)]. However, the skin depth is larger for Manganin also by virtue of its higher resistivity, which leads to a weaker electric field around the conductor and a reduced vortex current in the metal powder grains. Therefore, at high frequencies well above the cutoff frequency, filters with Manganin wires show a lower attenuation than copper wire filters. Although the majority of spectra confirm this behavior, the positive effect of the low skin depth of copper on the attenuation is small [see Fig. 8(c)]. Other parasitic effects, such as cross transmission as a result of the small winding distances and inhomogenities of the coil, are worse for $\mathrm{Cu}$ due to its lower skin-depth leading to a larger field around the conductor. This cross-transmission and the effect of the metal powder grain size largely outweigh this advantage of copper wires over Manganin.

\section{Powder type and grain size}

Technical grade metal powders are commercially available. However, their grain size and polydispersity are often unknown because the specified mesh size yields no information on the real averaged grain size (APS). Here we investigated the effect of powder grain size using copper $(\mathrm{Cu})$ and stainless steel (SS) powders with nominal mesh size in the range of -625 to -170 . The average grain size was determined by direct measurement in optical micrographs. According to earlier reports by Fukushima ${ }^{22}$ or Bladh, ${ }^{20}$ the optimal grain size is $30 \mu \mathrm{m}$ (-460 mesh) for $\mathrm{Cu}$ and $63 \mu \mathrm{m}$ (-243 mesh) for SS. In Figs. 7 and 8, the skin depth and typical attenuation curves of $\mathrm{SS}$ and $\mathrm{Cu}$ powders are shown. The copper powder $\mathrm{CuPA}^{50}$ (STREM Chemicals, USA) has an averaged grain size of about $10 \mu \mathrm{m}$ and the stainless steel powder SS-316L $\mathrm{L}^{51}$ (Alfa Aesar, Germany) has an averaged grain size of about $20 \mu \mathrm{m}$. The powders CuP1-5 $5^{52}$ and SS-316L were purchased at Alfa Aesar and used as received. The average particle size distribution was determined via direct measurements in a calibrated optical microscope via ImageJ software. In most cases, the nominal mesh size is larger than the measured median grain size of the metal powder. The properties of the powder used in this report are listed in Table I. SS-316L is a

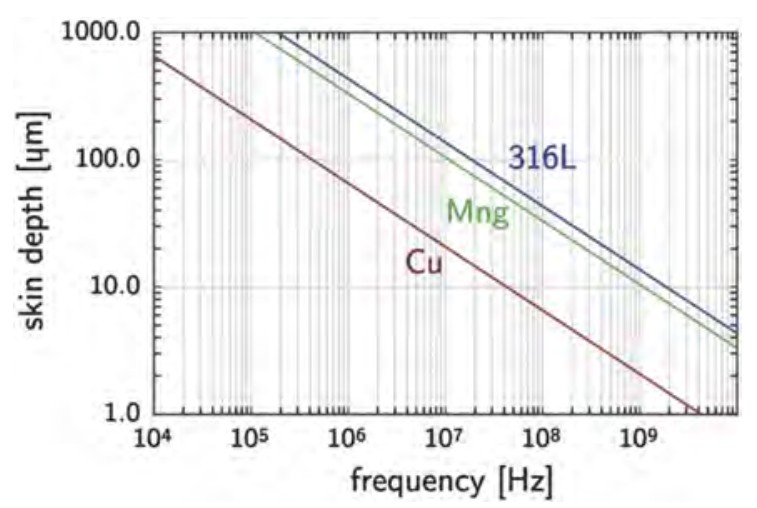

FIG. 7. Frequency dependent skin depth for copper $(\mathrm{Cu})$, stainless steel (316L), and Manganin (Mng). 

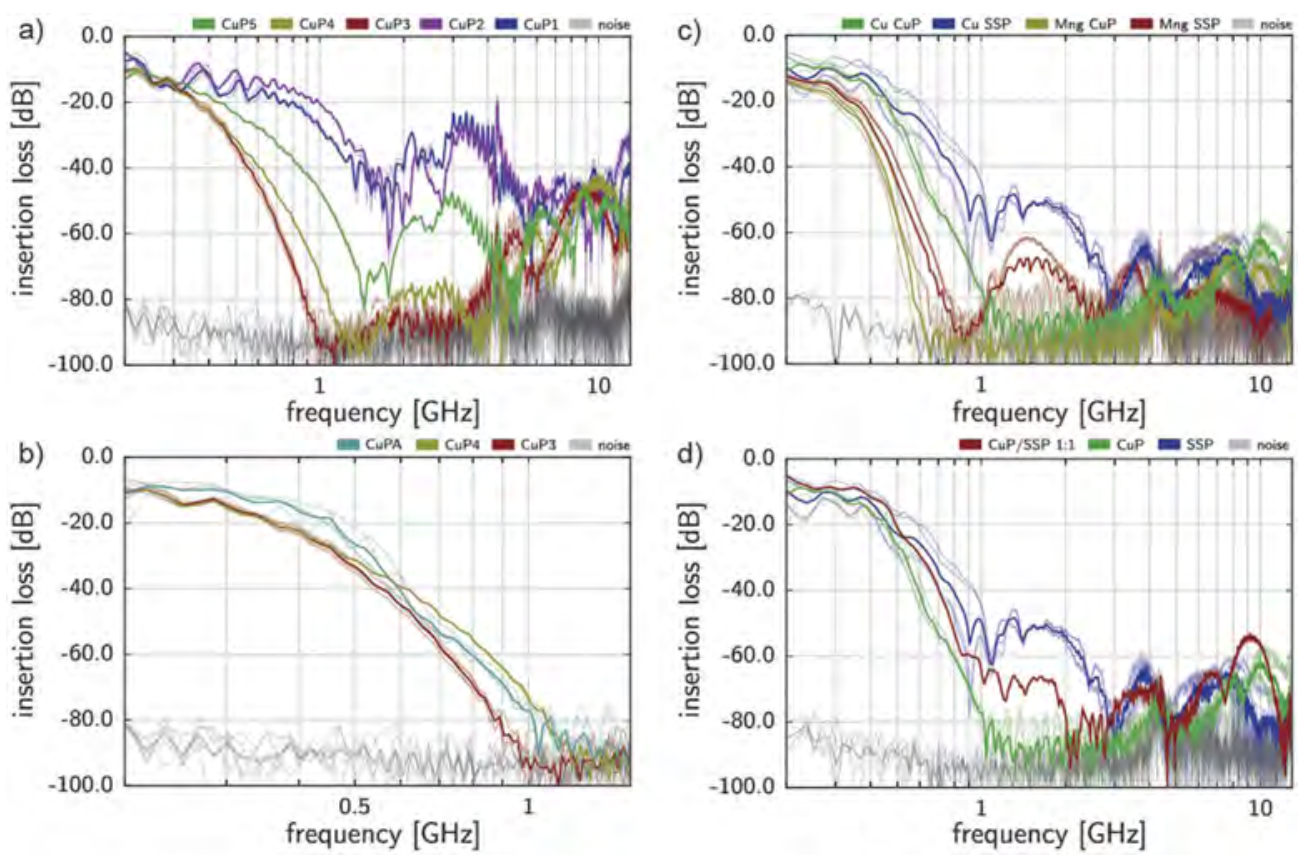

FIG. 8. (a) Comparison of different copper powders listed in Table I. CuP1 and CuP2 have a very small grain size with an arbitrary shape. CuP35 consists of grains with a spherical shape. The powder CuP5 has a very thin insulation sheet. The best performance shows CuP3 with average particle size (APS) of $10 \mu \mathrm{m}$. (b) Cutoff flank of different powders with different APSs and particle shapes. For powders with similar APS and low polydispersity, the flank is slightly steeper for the spherical grains than that for non-spherical powders, due to their higher packing density. (c) Transfer characteristics of powder filters made of different conductor and powder materials. (d) Comparison of stainless steel and copper powder filters with copper wires. A mixture of copper (CuPA) and steel (SS-316L) powders can be used to shift the frequency onset, where the damping is reduced to above $10 \mathrm{GHz}$.

stainless steel powder with an iron (III) oxyhydroxide layer. The others are copper powders with a copper (I) oxide layer with the exception of $\mathrm{CuP5}$, which was stored in a protective atmosphere with less than $1000 \mathrm{ppm}$ oxygen to prevent oxidation.

The properties of the metal powder affect the attenuation of the filter in multiple ways. First, Fukushima et $_{\text {al. }}{ }^{22}$ made an argument based on the packing density. If it is too high, the effective surface on which the skin effect acts becomes small and the attenuation is reduced. This case is shown in Fig. 8(a) in the measurement of powders $\mathrm{CuP} 1$ and $\mathrm{CuP} 2$, which have a very small grain size. However, both $\mathrm{Cu}$ and $\mathrm{SS}$ powders usually have a native oxide layer, which is insulating and separates the particles electrically. A reduction of the effective surface area only occurs, when the oxide insulation is broken or bare metal powder is used. We found that even after ultrasonication for several minutes to compact the powder in the coil, the oxide layer remains intact and the attenuation behavior remains unchanged. The importance of the insulation sheet can be seen

TABLE I. Properties of investigated metal powders.

\begin{tabular}{lccc}
\hline \hline Name & Mesh & APS $(\mu \mathrm{m})$ & Shape \\
\hline CuPA & -325 & 10 & Non-spherical \\
SS-316L & -325 & 20 & Non-spherical \\
CuP1 & -625 & $0.5-1.5$ & Non-spherical \\
CuP2 & -625 & $2.0-3.5$ & Non-spherical \\
CuP3 & -375 & 10 & Spherical \\
CuP4 & $-170+270$ & 70 & Spherical \\
CuP5 & $-170+400$ & 50 & Spherical \\
\hline \hline
\end{tabular}

in the performance of $\mathrm{CuP5}$ (Fig. 8). This powder features an optimal grain size like CuP3 and CuP4, but it was kept in a protective atmosphere with less than $1000 \mathrm{ppm}$ oxygen. As a result, the native oxide layer cannot form and the bare metal shows a weak attenuation compared to $\mathrm{CuP} 3$ and CuP4. Essentially, the use of slightly polydisperse powders, such as $\mathrm{CuP3}$, can improve the filter characteristics by increasing the packing density of the powder, although the mean particle diameter is below the optimal value. The second argument considers the skin depth of the powder material. If the skin depth is smaller than or comparable to the particle radius, the vortex current cannot be fully established. Hence, at this point, the attenuation is reduced, such as shown in Fig. 8(a) for filters made of $\mathrm{CuP} 1$ and CuP2 and in Figs. 8(c) and 8(d) for filters with SSP (SS-316L) in the range of 1 until $5 \mathrm{GHz}$. The measurements of SS powder filters show a lower attenuation at low frequencies compared to copper powder filters. The reason is that the skin depth of stainless steel reaches the averaged grain radius of $10 \mu \mathrm{m}$ at a frequency of approximately $2 \mathrm{GHz}$, where a reduction of the attenuation by more than $20 \mathrm{~dB}$ compared to $\mathrm{CuP} 4$ is observed. For most copper powders, the average particle radius is much larger than the skin depth at frequencies above $120 \mathrm{MHz}$ and thus has no influence on the attenuation in the relevant frequency range analyzed in Fig. 8(b).

The influence of different metal powders in combination with both copper and Manganin wires is illustrated in Fig. 8(c). The attenuation of filters built with Manganin wires improves drastically at frequencies between $700 \mathrm{MHz}$ and $1 \mathrm{GHz}$ due to its higher resistivity compared to filters with copper wires. On the other hand, since Manganin has a higher skin depth than copper, the field around the conductor is weaker, which 
leads to the reduced attenuation at high frequencies. Hence, Manganin wire filters show a steep cutoff but an inferior performance at frequencies above 3-5 GHz. Regarding the metal powder, at high frequencies above $5 \mathrm{GHz}$, the much larger skin-depth of SS-316L compared to copper powder yields a higher insertion loss irrespective of the wire type. However, these filters constructed with SS-316L powder [red and blue traces in Fig. 8(c)] show a significantly reduced attenuation at intermediate frequencies between $700 \mathrm{MHz}$ and $3 \mathrm{GHz}$, where the skin-depth is in the order of the particle size. In this frequency range, the higher resistivity of the Manganin wire partially compensates the negative effect of the powder material on the filter performance, which results in a $-20 \mathrm{~dB}$ higher insertion loss compared to copper wire filters. Altogether engineering the performance of metal powder filters requires the detailed consideration of many different material- and design parameters, which can be optimized to yield an insertion loss well above $-80 \mathrm{~dB}$ at intermediate frequencies. However, a major drawback of all powder filters is their tendency to reduced attenuation at high frequencies above 7-10 GHz, depending on the design, when the electromagnetic wave is transmitted through the linear filter coil from one winding layer to the next. The specific characteristics above this frequency range are mostly unpredictable and depend on the powder, the wire type, and its dimensions, but to a large extent also on very specific details of the actual filter coil, its geometry, winding distance, and distortions. In Fig. 8(d), we compare filters with a copper coil in combination with CuPA, SS-316L, and a CuPA/SS-316L mixed powder. Great care was taken to achieve identical coils with as little variation in the geometry as possible. All three filters show a characteristic trend towards reduced attenuation at high frequencies. However, the onset of the damping reduction depends on the type of the powder material, which is around $8-13 \mathrm{GHz}$ for $\mathrm{CuPA}$ and 5-9 GHz for the SS-316L powder. The onset of the filter with $50 / 50$ powder mix is perfectly between the other two measurements around 7 to $11 \mathrm{GHz}$, and the damping reduction also scales with the mixing ratio between $\mathrm{CuPA}$ and SSP-316L. However, regardless of the wire type, powder material, and filter construction, all metal powder filters are prone to drastically reduced HF attenuation at frequencies above $10 \mathrm{GHz}$. Therefore, they have to be complemented by other types of filters with better performance at high frequencies.

\section{FERRITE COMPOUND FILTERS}

In order to address the shortcomings of powder filters illustrated above, we investigate a novel transmission line filter type based on two unique features. First, the wound wire coil or powder filters are replaced by a transmission line, representing a distributed filter element that can be micropatterned on a PCB with very high accuracy and reproducibility. Second, we employ a magnetically lossy medium (ferrite powder compound) that provides a higher loss tangent compared to the non-magnetic powders employed in the fabrication of powder filters above. Two different circuit designs were tested: a simple stripline and a structured transmission line "harp" structure. For each design, we tested both hard and soft
TABLE II. Properties of ferrite powders used in absorber compounds.

\begin{tabular}{lclc}
\hline \hline Powder & Grain size $(\mu \mathrm{m})$ & Material & $\begin{array}{l}\text { Type hard/ } \\
\text { soft ferrite }\end{array}$ \\
\hline OP-71 & $1-2$ & Strontium ferrite & Hard \\
OP-56 & $0.5-1.5$ & Strontium ferrite & Hard \\
UF-S1 & $<0.6$ & Strontium ferrite & Hard \\
3S3 I 3S3a & $90-200 \mid 10-30$ & Manganese zinc & Soft \\
TDW & $20-30$ & Manganese zinc & Soft \\
MCS compound & $1.5 \mid 25-60$ & Not specified & Soft \\
GDS compound & $1.5 \mid 10-50$ & Not specified & Soft \\
\hline \hline
\end{tabular}

ferrites. For testing, three strontium ferrites ${ }^{53}$ (OP-71, OP-56 and UF-S1) from the manufacturer Dowa F-TEC Co Ltd., and two manganese zinc ferrites, named $3 \mathrm{~S}^{54}$ and $\mathrm{TDW}^{55}$ from the companies Tropag GmbH and Tridelta Weichferrite $\mathrm{GmbH}$, were mixed with liquid silicone rubber and cast into flexible sheets of absorber material. The properties of the powders are summarized below in Table II. For comparison, we also tested commercially available ferrite compounds, labeled MCS and GDS, from Emerson and Cuming, which are composed of at least two different metal powders with small, spherical grains and large irregularly shaped soft-ferrite grains.

A comparison of the characteristics of stripline filters with these ferrite compounds is shown in Fig. 9. It is clear that hard ferrites, due to their high coercivity, are too stiff to produce a large magnetic loss component for high frequency signals. Therefore these compounds cannot provide as good low-pass filtering as soft ferrites at all frequencies. Moreover, the grain size of the hard ferrites is well below $10 \mu \mathrm{m}$, which is too small to yield a significant loss due to the skin effect, as discussed in Sec. V for non-magnetic powder filters.

As shown in Fig. 9, a matched stripline structure, i.e., a short wire, does not show pronounced resonances in the

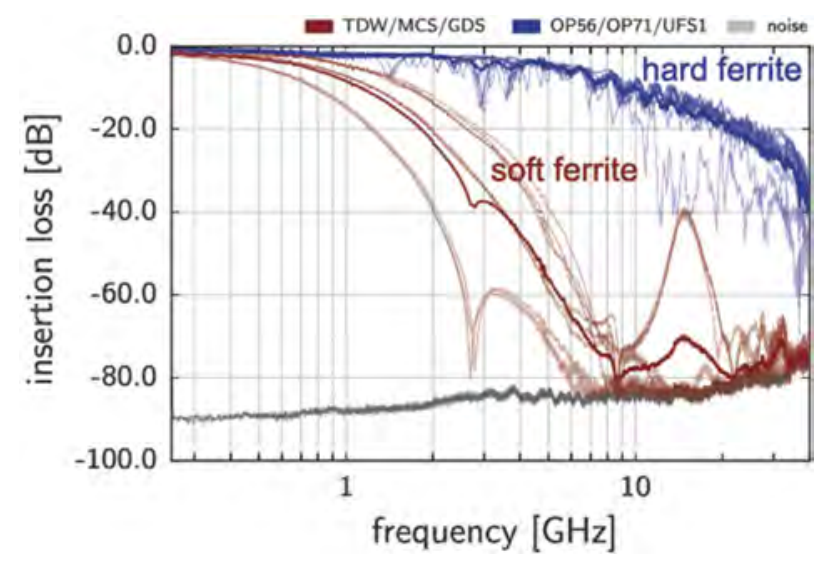

FIG. 9. Comparison of the attenuation spectrum of hard ferrites (OP56, OP71, UFS1) and soft ferrites (TDW, MCS, GDS) in a stripline filter geometry. The coercivity of the hard ferrites is large and the grain size of this powder is too small to yield considerable attenuation, while the large magnetic loss tangent soft ferrites provides an additional dissipation channel leading to much better attenuation. 
transmission spectrum and produces a weak low-pass characteristic, even with a magnetically loaded absorber material. Therefore, here we develop a "harped" filter structure, which is a micropatterned transmission line with multiple vertical pads. This geometry shows several resonances and yields a steep flank in the attenuation spectrum, which allows us to construct a filter with well-defined low-pass characteristics. Examples of harped transmission lines with different geometries are shown in Fig. 10(a). Each pad length corresponds to a certain resonance observed in the transmission spectrum and

a)

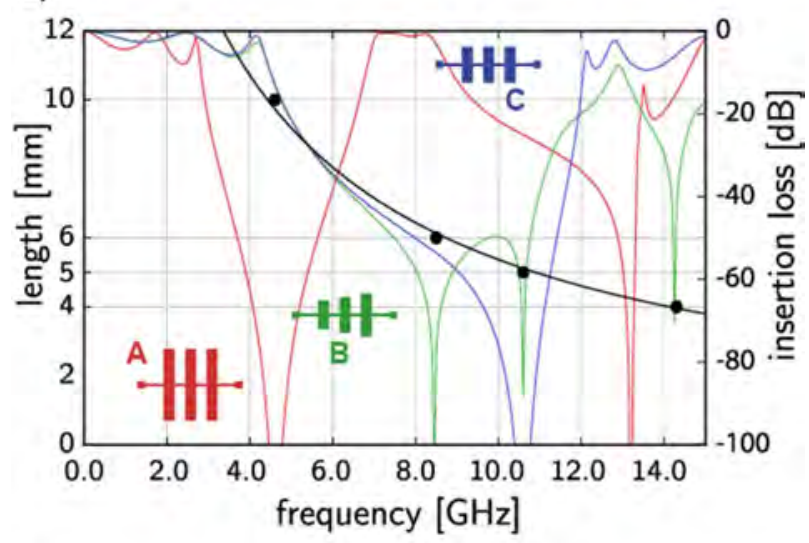

b)

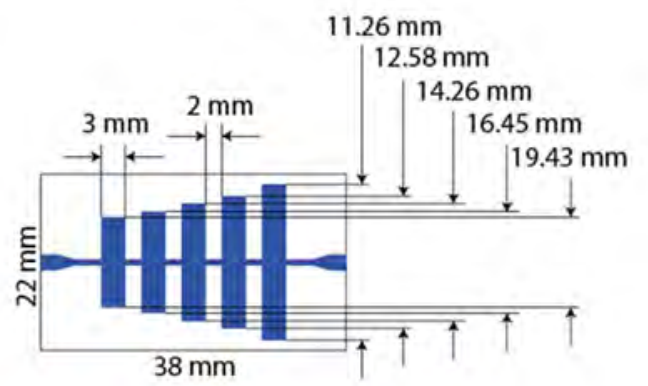

c)

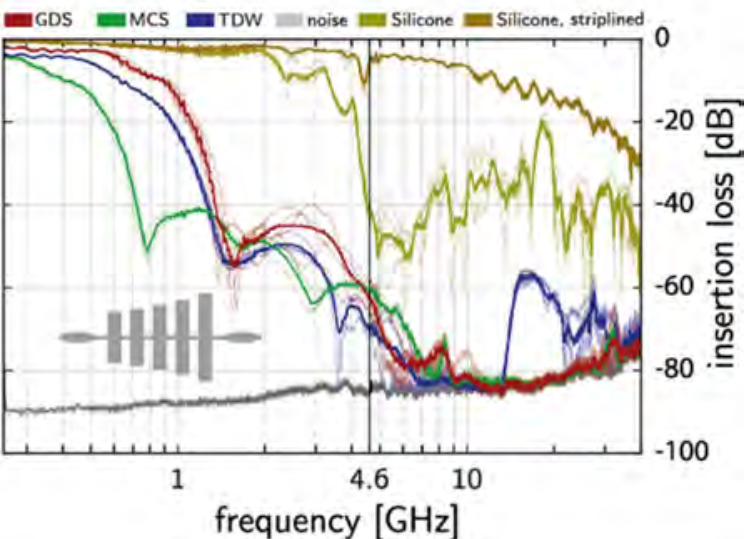

FIG. 10. (a) Sonnet spectral simulation of three different harp structures with a pad length of (A) 10-10-10 mm, (B) 4-5-6 mm, (C) 5-5-5 mm. Each resonance in the transmission spectrum corresponds to a certain pad length (black circles). The resonance frequency of the pads is reciprocal to their size and thus enables the design of low-pass filters with tailored filter characteristics. (b) Sketch of the final harp structure used in subsequent measurements. (c) Measurements of the two commercial ferrite compounds GDS and MCS and the self-produced silicone compound TDW. The yellow spectrum is a measurement of the pristine silicone matrix without metal ferrite powder using the stripline geometry (dark yellow) and harped transmission line (light yellow). is well described by theory. ${ }^{56}$ In order to conceptualize a filter, we investigated several harp designs in a 2D and 3D HF electromagnetic simulation (Sonnet EM Solver) and obtained well-distributed resonances over the spectral range between 2 and $10 \mathrm{GHz}$. The dependence of the resonance frequency and the pad length is reciprocal and can be evaluated empirically through a simple fit function $v(L)=\frac{c}{L}+\delta v$ [see Fig. 10(a)]. Based on these simulations, we designed the harped structure [Fig. 10(b)], which consists of a transmission line featuring five pads with a width of $3 \mathrm{~mm}$ and pad lengths of 19.43, $16.45,14.26,12.58$, and $11.26 \mathrm{~mm}$, respectively, corresponding to resonances at frequencies of 4.6, 5.8, 7.0, 8.2, and $9.4 \mathrm{GHz}$. The improvement of this harp structure in comparison to a simple stripline is shown in Fig. 10(c). The insertion loss of the harped structure increases dramatically around the first resonance at $4.6 \mathrm{GHz}$ to about $-50 \mathrm{~dB}$, while the simple stripline yields a weak damping below $-10 \mathrm{~dB}$.

In order to construct a low-pass filter, the optimized harp structure [Fig. 10(b)] is patterned on a thin PCB and embedded in a lossy medium. Based on the considerations above, we investigated the absorption behavior of different ferrite compounds. Again, the grain size of the ferrite powder is important to obtain a good attenuation across the entire spectrum. The as-received $3 \mathrm{~S} 3$ powder showed a grain size in the range of $90-200 \mu \mathrm{m}$, which is too large to achieve a sufficiently high packing density in the compound necessary to produce the desired filtering characteristics. Grinding the material again with a simple mortar yields a powder (3S3a) with average grain size in the range of $20 \mu \mathrm{m}$, comparable to TDW. However, the particle size distribution and particle shape became irregular after grinding, especially a large fraction of small particles is observed, which results in a very high packing density and therefore non-optimal damping performance (not shown).

As discussed above, besides the grain size, the powder density in a ferrite compound is an important quantity determining the overall filter characteristics. However, to further improve the filter properties, we investigate filters, where the lossy absorber material is a rubbery compound incorporating the metal powder. The advantage of such compounds is their better handling, usability, and stability, the lower risk of electrical shortcuts and the omission of a hermetic enclosure. Excellent broad-band low pass characteristics are obtained, when the harped transmission line is embedded in a magnetically lossy ferrite absorber compound. However, while the transfer function of the harped transmission line itself can be calculated theoretically, when cross-currents are negligible, there is no adequate description of the filter characteristics in the presence of the metal-loaded absorber compound. A detailed discussion on a triplate geometry, where a transmission line is sandwiched between two conducting layers can be found in Ref. 56.

Three different weight ratios of ferrite powder and nonoutgassing silicone rubber are analyzed (4:1, 3:1, 2:1). As expected, the compound with higher powder loading showed a much better damping performance; Figs. 11(a) and 11(b) display the transmission spectra of simple stripline and harped transmission line filters with TDW compounds at different loading fractions. In principle, it would be possible to increase 

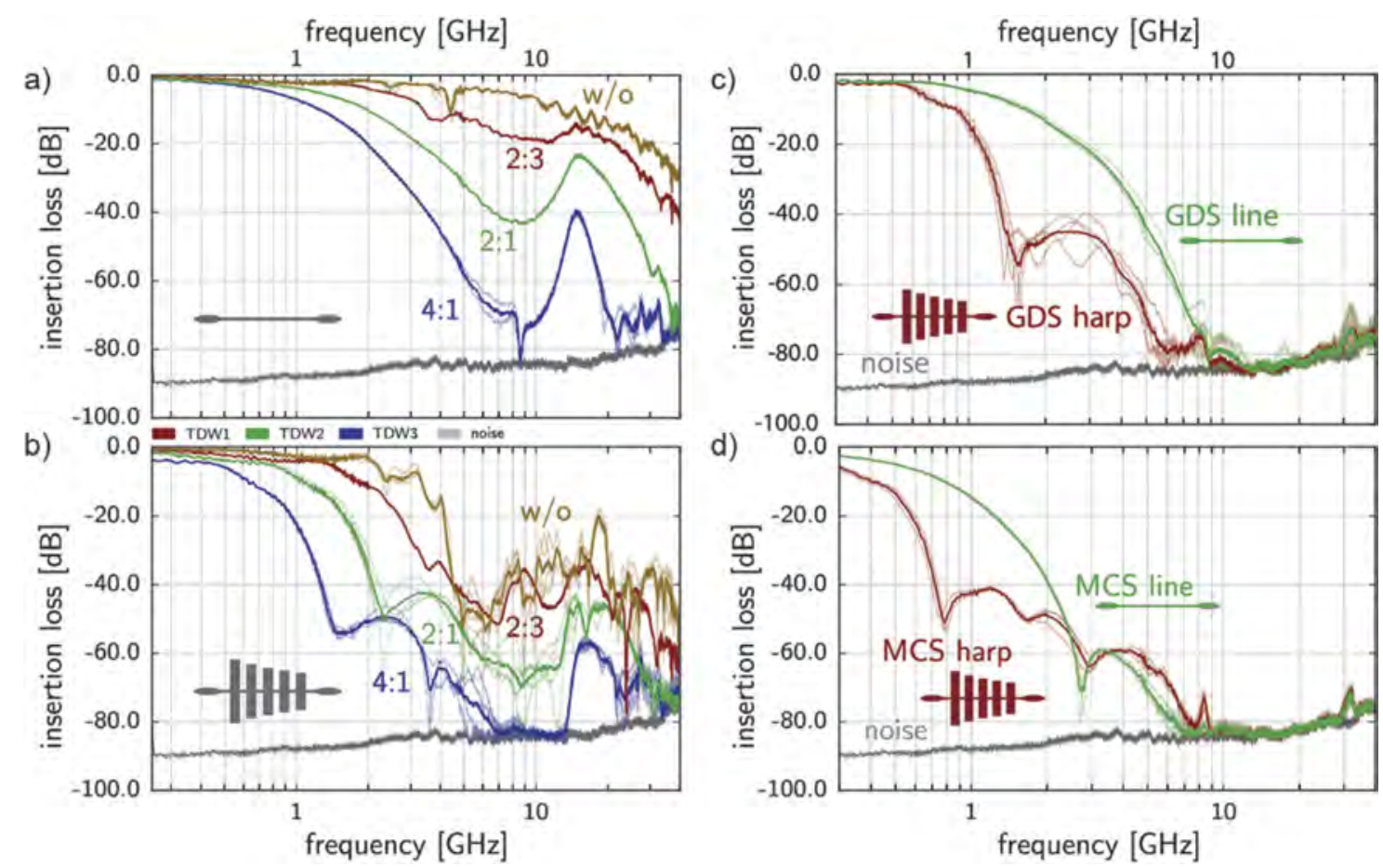

FIG. 11. (a) Three different powder densities in the compounds are measured with the soft ferrite powder TDW in a stripline geometry. The fractions indicate the weight ratio of powder and silicone rubber. (b) The weak attenuation across the entire spectrum can be improved by the harp structure and at high metal contents (4:1) becomes comparable to commercially available absorber compounds. This compound in combination with harped filter produces an attenuation of more than $-56 \mathrm{~dB}$ already at the lowest resonance around $1.5 \mathrm{GHz}$. (c) The spectrum of the compound GDS shows a steeper flank than MCS and maintains a strong attenuation after reaching the noise level at $9 \mathrm{GHz}$ even in the stripline geometry. (d) The spectrum of MCS reaching the noise level at $7 \mathrm{GHz}$ with no reduction of attenuation up to $40 \mathrm{GHz}$. Notably, above $2.6 \mathrm{GHz}$, the performances of the stripline and harped filter are very similar.

the fraction of metal powder further; however, the compound is then hard to mix and becomes stiff and inhomogeneous, which results in a lower attenuation. Compared to the custommade absorber compound, the commercially available alternative incorporates the soft ferrite powder in a matrix of small spherical, non-magnetic particles and a rubbery component. Apparently, this mixture provides better flexibility while maintaining excellent damping characteristics shown in Figs. 11(c) and $11(\mathrm{~d})$.

The filters with the ferrite powder TDW [see Figs. 11(a) and 11(b)] show a pronounced decrease of the attenuation around $15 \mathrm{GHz}$. This unwanted decrease can be counteracted by using a harp structure as shown in Fig. 11(b). Here a harp was designed that provides a strong damping at $13 \mathrm{GHz}$ and suppresses the unwanted decrease of the attenuation by more than $-20 \mathrm{~dB}$ although it is still visible in the spectra. Moreover, the spectra show that a high insertion loss below the noise floor of our setup can be achieved at frequencies above $25 \mathrm{GHz}$ by finding the optimal composition of the absorber compound. Moreover, the harped transmission line also improves the attenuation between $0.5 \mathrm{GHz}$ and $8 \mathrm{GHz}$ compared to the stripline geometry. Notably at the first resonance around $1.5 \mathrm{GHz}$, a damping better than $-56 \mathrm{~dB}$ is achieved. As shown in Figs. 11(c) and 11(d), two other well working compounds are the commercial MCS and GDS absorbers. The MCS compound has a flatter flank and shows a better lowpass characteristic in a range of $1-10 \mathrm{GHz}$ but it is known to be more sensitive to temperature changes than GDS. ${ }^{29}$ In our filter design, the attenuation with both the GDS and MCS compounds reach the noise level at about $10 \mathrm{GHz}$ (e.g., -86 $\mathrm{dBm}$ with a resolution bandwidth of $510 \mathrm{kHz}$ in our measurements) with no reduction at higher frequencies up to $40 \mathrm{GHz}$. Moreover, the spectra once again show the positive effect of the harp structure in the filter characteristics compared to the stripline filter, which results in a much steeper flank at $1 \mathrm{GHz}$ or even below, depending on the pad design and the absorber used.

Parasitic resonances can form in the microstripline, due to field distortions within the structure as a result of irregularities and the effect of the electromagnetic environment. This can be seen clearly at $2.6 \mathrm{GHz}$ in the measurements of MCS [Fig. 11(d)] and less pronounced in the measurements of GDS [Fig. 11(c)] at $8.8 \mathrm{GHz}$. These resonances can be understood considering the high permittivity of this compound so that the resonance frequency for the stripline is below the noise-levelcut-off frequency. The effective permittivity at the first mode for a resonator with ideal and stray-field free ends and a length $l$ at frequency $v$ is given by Ref. $56 \sqrt{\varepsilon_{\text {eff }}}=\frac{c}{2 l v}$, which yields a permittivity of about 2.3 for MCS and 0.2 for GDS. This shows that the properties of the absorber compound need to be matched with the frequency response of the harped filter structure to obtain optimal low-pass characteristics. Nevertheless, the overall filter response is to a large extent determined by the distributed elements transmission line structure and its specific design.

Besides the above named benefits, the concept of lowpass filters with metal-loaded absorber compounds has several advantages over metal powder filters, as it can be used to 

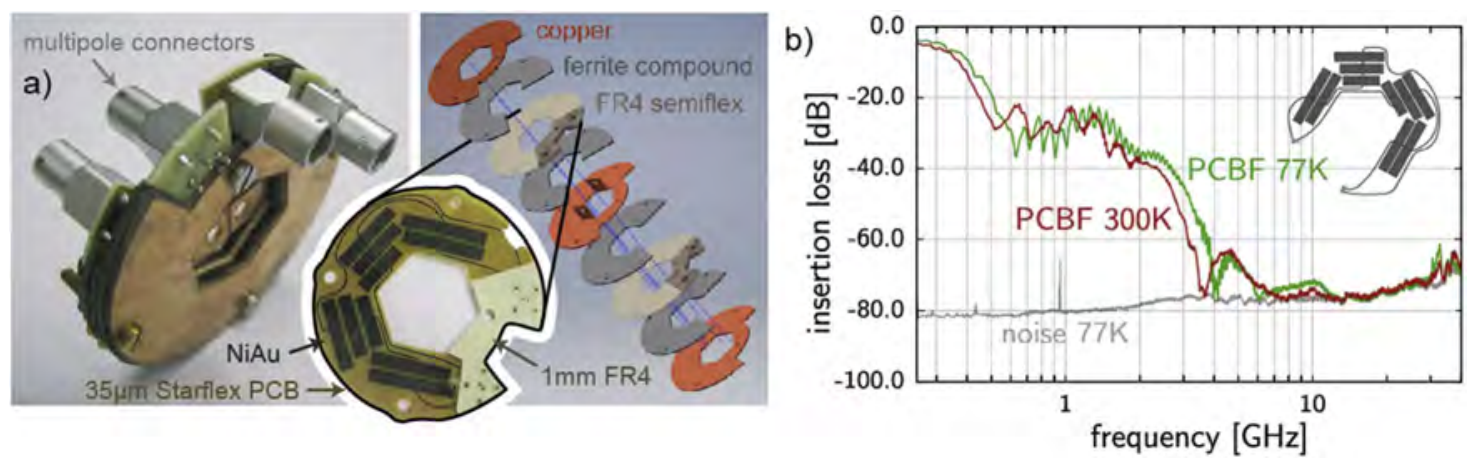

FIG. 12. Design of ferrite compound filters. (a) The filter board is sandwiched between the absorber compounds and shaped to fit over a hexagonal thermalization rod mounted at the mixing chamber of the cryostat. (b) Attenuation spectrum of the low-pass filter shown in (a) at bath temperatures of $77 \mathrm{~K}$ and at $300 \mathrm{~K}$.

design complex filter characteristics for single- and multipole filters on a PCB even in space-constraint cryogenic assemblies, which are very easy to manufacture. Figure 12 shows an example of how such filters can be implemented in a dilution refrigerator at base temperature. The assembly consists of one or multiple PCBs with two lines, each incorporating a harped transmission line as distributed filter element, sandwiched between the absorber compounds. This compact filter is mechanically and thermally anchored in the cryostat using a simple copper fitting. It is important to note that the concept itself can be easily adopted to other filter geometries and allows construction of compact multi-channel filters with well-defined filter characteristics. However, the performance of these filters relying on a magnetically lossy environment is sensitive to external magnetic fields. A sufficiently large magnetic DC field will saturate the ferrite compound, which results in a reduction of the magnetic loss tangent and a notable increase of the cutoff frequency. Therefore, ferritecompound filters should be backed by other field-insensitive filters in applications, where large external magnetic fields used.

In the example shown in Fig. 12, we used a transmission line filter with five pads located in two groups for a two-channel measurement line. The filter characteristics show a sharp cutoff at $200 \mathrm{MHz}$ and an insertion loss of more than $-60 \mathrm{~dB}$ at frequencies above $3 \mathrm{GHz}$, while the noise floor is reached at $5 \mathrm{GHz}$. In combination with standard RC filters or the capillary wire filters shown above, very neat low-pass filter characteristics can be achieved. Moreover, measurements at liquid nitrogen temperature $(77 \mathrm{~K}$ ) showed that the attenuation spectrum varies only slightly with temperature. Yet another advantage of these lossy transmission line filter elements resides in the fact that the insertion loss is almost independent of the termination load and hence a versatile filter for different measurement tasks can be designed.

The combined filter characteristics of a twisted pair measurement line incorporating a capillary filter and a ferrite compound filter is shown in Fig. 13. The functionality of the filter combinations has been tested in several quantum transport experiments in superconducting circuits as well as on atomic-scale metal- and molecular contacts and spintronic nanodevices. As first example multiple Andreev reflections (MARs) in a superconducting aluminum single atom, contacts were measured at a base temperature around $25 \mathrm{mK}$. MARs are multi-particle charge transport processes that set in at voltages of $e V=\frac{2}{n} \Delta$, where $n$ is the number of electrons transported in a process and $\Delta$ is the superconducting gap $(\Delta=187 \mu \mathrm{eV})$ in aluminum. The MAR effect gives rise to non-linear current-voltage-characteristics, which results in a series of well-defined maxima and minima in the differential conductance $\mathrm{d} I / \mathrm{d} V$ of the sample (not shown). The observation of MAR up to the 6th order confirms the excellent noise suppression and reduction of the electronic temperature by using the low-pass filter concept introduced in this report. Although these measurements do not allow extraction of the effective electronic temperature of the system, the observed MAR spectra can be fitted using the zero-temperature model of MARs. Similarly, the observation of Shapiro steps in a Josephson junction of $\mathrm{Al}$ under microwave irradiation, shown in Fig. 13(c), confirms the excellent noise suppression. Shapiro steps are replica of the supercurrent branch and appear in current-biased Josephson junctions as steps in the IV characteristics below
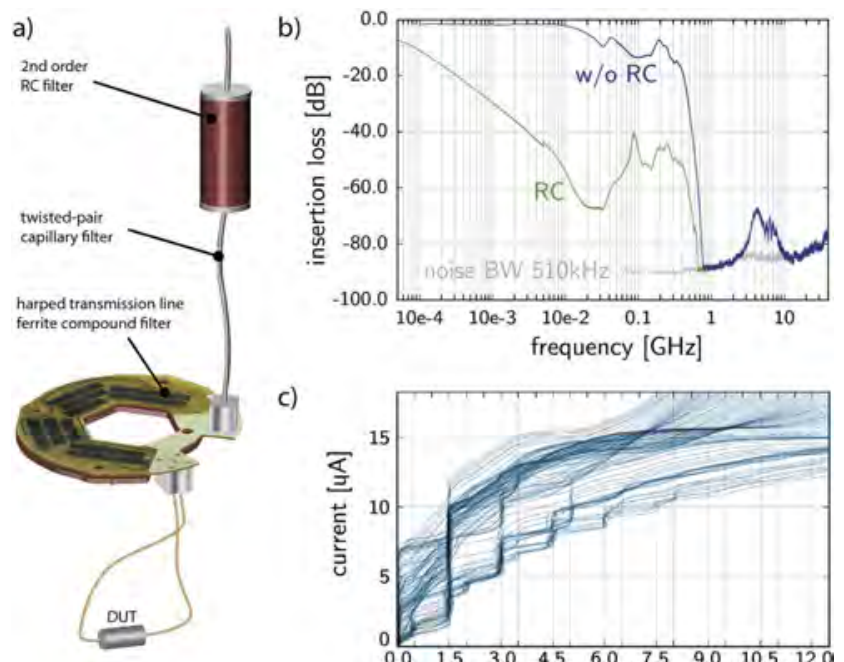

c)

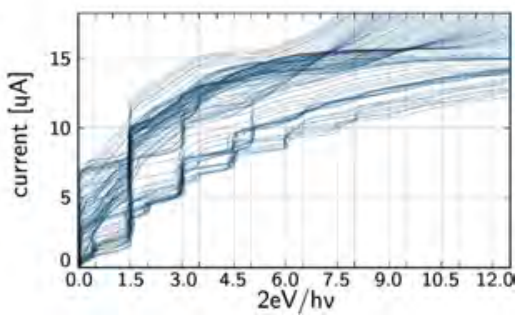

FIG. 13. (a) Sketch of a filtering scheme combining several types of low-pass filters with different attenuation characteristics. (b) Spectra of a series combination of a 2nd order RC-filter, a $1 \mathrm{~m}$ long ES shielded capillary filter, and a MCS-PCB filter. (c) Shapiro steps in the current-voltage characteristics of an aluminum Josephson junction under HF irradiation at different frequencies from $0.5 \mathrm{GHz}$ to $30 \mathrm{GHz}$ at a constant $\mathrm{HF}$ power of $-76 \mathrm{dBm}$ at the sample. 
$\Delta$ at voltage intervals of $\Delta V=\frac{\hbar \omega}{2 e}$, where $\omega$ is the microwave frequency and $e$ is the electron charge. The observation of welldeveloped Shapiro steps with a separation of $\Delta \mathrm{V}=2 \mu \mathrm{V}$ at low $\mathrm{HF}$ frequencies of $f=1 \mathrm{GHz}$ demonstrates the improvement of energy resolution of the measurement circuit below $2 \mu \mathrm{V}$, which corresponds to $\mathrm{k}_{\mathrm{B}} \mathrm{T}=2 \mu \mathrm{V}$ that corresponds to a temperature of roughly $23 \mathrm{mK}$ and is below the bath temperature of $30 \mathrm{mK}$. This improvement of energy resolution is largely due to the suppression of HF input noise to the sample stage and in the DC measurement lines. However, both MAR and Shapiro measurements do not provide direct access to the effective electronic temperature of the system. The latter can be determined via primary thermometry, including the analysis of the Johnson-Nyquist ${ }^{57,58}$ or shot-noise ${ }^{59}$ of a calibrated sample resistor or Coulomb blockade spectroscopy ${ }^{3,30,60}$ among other techniques, ${ }^{61,62}$ which can be used to determine the absolute sample temperature.

\section{SUMMARY AND CONCLUSION}

In this report, we compare different types of cryogenic low-pass filters including RC filters, capillary filters, copper powder filters, and ferrite compound filters; each of them has its own advantages and disadvantages along with a characteristic transmission spectrum with low-pass cutoff at different frequencies. For each filter type, we introduce a robust design that can be easily adapted to other measurement applications. The frequency response of the filters can be tuned by various means to produce the required filter characteristics and the combination of several filters with different concepts shows excellent, tunable low-pass filter characteristics with nearly ideally sharp cutoff at a designable frequency (typically below $1 \mathrm{GHz}$ ), suitable for low-temperature quantum transport measurements.

Many transport experiments reported so far rely on a single type of filters, namely, metal powder filters. However, typically metal powder filters show good low-pass characteristic only if the relevant design parameters are very well defined, otherwise their filter characteristic becomes non-monotonous and irregular, especially at higher frequency range around 10$20 \mathrm{GHz}$, where a reduced attenuation is frequently observed. Because this filter type is very sensitive to small changes in the winding distances, to variations in the packing density and to coil inhomogeneities, the filter characteristics are hard to predict or reproduce. However, due to the encapsulation of the measurement leads in metal powder, these filters may serve as reliable thermal anchors at different temperature stages in a cryogenic environment. In contrast to the metal powder filters, the new ferrite compound filters based on distributed transmission line elements can be fabricated accurately and their spectral characteristics are easy to define and reproducible. Moreover, this compact filter type is very easy to fabricate in different geometries and can be used in space constraint cryogenic setups. With optimized composition of the absorber compound, excellent high frequency rejection is achieved above $4 \mathrm{GHz}$ up to at least $40 \mathrm{GHz}$. The design of distributed, harped elements also improves the performance at frequencies below $1 \mathrm{GHz}$.
The combination of at least two different filter types (out of RC filters, capillary filters, powder filters and compound filters) yields an excellent attenuation over the entire frequency range from $\mathrm{kHz}$ range up to several tens of GHz. Here, capillary RC filters provide low-pass filtering below $1 \mathrm{GHz}$, while copper or stainless steel powder filters are suitable for a frequency range from $1 \mathrm{GHz}$ to $6 \mathrm{GHz}$ and also provide the thermal anchoring of the measurement leads. The distributed elements ferrite compound filter introduced in this article very efficiently removes high frequency components above $5 \mathrm{GHz}$. Hence, a simple setup incorporating only capillary, RC and compound filters, already provides sufficient reduction of the electronic temperature and noise rejection to conduct quantum transport measurements with an energy resolution below the base temperature of the setup at $25 \mathrm{mK}$.

\section{ACKNOWLEDGMENTS}

We thank C. Schirm, C. Debuschewitz, and T. Geiger for their contributions in the early phase of the project and for fruitful discussions on the implementation and description of different low-pass filters. T. Pietsch gratefully acknowledges financial support from the Zukunftskolleg of the University of Konstanz.

${ }^{1}$ S. M. Goodnick and J. Bird, IEEE Trans. Nanotechnol. 2, 368 (2003).

${ }^{2}$ K. K. Likharev, Proc. IEEE 87, 606 (1999).

${ }^{3}$ J. P. Pekola, O. P. Saira, V. F. Maisi, A. Kemppinen, M. Möttönen, Y. A. Pashkin, and D. V. Averin, Rev. Mod. Phys. 85, 1421 (2013).

${ }^{4}$ J. Clarke and F. K. Wilhelm, Nature 453, 1031 (2008).

${ }^{5}$ M. H. Devoret and R. J. Schoelkopf, Science 339, 1169 (2013).

${ }^{6}$ M. H. Devoret, A. Wallraff, and J. M. Martinis, e-print arXiv:condmat/0411174 (2004).

${ }^{7}$ H. F. Pernau, T. Pietsch, and E. Scheer, J. Phys.: Condens. Matter 26, 474203 (2014).

${ }^{8}$ T. Pietsch, S. Egle, M. Keller, H. Fridtjof-Pernau, F. Strigl, and E. Scheer, New J. Phys. 18, 093045 (2016).

${ }^{9}$ E. Scheer, T. Böehler, A. Edtbauer, S. Egle, A. Erbe, and T. Pietsch, Low Temp. Phys. 39, 259 (2013).

${ }^{10}$ H. Bluhm, S. Foletti, I. Neder, M. Rudner, D. Mahalu, V. Umansky, and A. Yacoby, Nat. Phys. 7, 109 (2011).

${ }^{11}$ B. Braunecker, P. Simon, and D. Loss, Phys. Rev. B 80, 165119 (2009).

${ }^{12}$ J. R. Petta, A. C. Johnson, J. M. Taylor, E. A. Laird, A. Yacoby, M. D. Lukin, C. M. Marcus, M. P. Hanson, and A. C. Gossard, Science 309, 2180 (2005).

${ }^{13}$ C. P. Scheller, T. M. Liu, G. Barak, A. Yacoby, L. N. Pfeiffer, K. W. West, and D. M. Zumbühl, Phys. Rev. Lett. 112, 066801 (2014).

${ }^{14}$ P. Simon and D. Loss, Phys. Rev. Lett. 98, 156401 (2007).

${ }^{15}$ R. Takahashi, K. Kono, S. Tarucha, and K. Ono, Phys. Rev. Lett. 107, 026602 (2011).

${ }^{16}$ V. A. Mikheev, P. G. Noonan, A. J. Adams, R. W. Bateman, and T. J. Foster, Low Temp. Phys. 34, 404 (2008).

${ }^{17}$ W. Frost, Heat Transfer at Low Temperatures (Springer US, New York, 1975).

${ }^{18}$ F. Pobell, Matter and Methods at Low Temperatures (Springer, Heidelberg, Berlin, 2007).

${ }^{19}$ D. C. Glattli, P. Jacques, A. Kumar, P. Pari, and L. Saminadayar, J. Appl. Phys. 81, 7350 (1997).

${ }^{20}$ K. Bladh, D. Gunnarsson, E. Hürfeld, S. Devi, C. Kristoffersson, B. Smålander, S. Pehrson, T. Claeson, P. Delsing, and M. Taslakov, Rev. Sci. Instrum. 74, 1323 (2003).

${ }^{21}$ M. M. Freund, T. Hirao, V. Hristov, S. Chegwidden, T. Matsumoto, and A. E. Lange, Rev. Sci. Instrum. 66, 2638 (1995).

${ }^{22}$ A. Fukushima, A. Sato, A. Iwasa, Y. Nakamura, T. Komatsuzaki, and Y. Sakamoto, IEEE Trans. Instrum. Meas. 46, 289 (1997).

${ }^{23}$ A. Lukashenko and A. V. Ustinov, Rev. Sci. Instrum. 79, 014701 (2008).

${ }^{24}$ F. P. Milliken, J. R. Rozen, G. A. Keefe, and R. H. Koch, Rev. Sci. Instrum. 78, 024701 (2007). 
${ }^{25}$ F. Mueller, R. N. Schouten, M. Brauns, T. Gang, W. H. Lim, N. S. Lai, A. S. Dzurak, W. G. van der Wiel, and F. A. Zwanenburg, Rev. Sci. Instrum. 84, 044706 (2013).

${ }^{26}$ J. M. Martinis, M. H. Devoret, and J. Clarke, Phys. Rev. B 35, 4682 (1987).

${ }^{27}$ H. Bluhm and K. A. Moler, Rev. Sci. Instrum. 79, 014703 (2008).

${ }^{28}$ A. Kushino and S. Kasai, J. Supercond. Novel Magn. 28, 715 (2015).

${ }^{29}$ D. F. Santavicca and D. E. Prober, Meas. Sci. Technol. 19, 087001 (2008).

${ }^{30}$ C. P. Scheller, S. Heizmann, K. Bedner, D. Giss, M. Meschke, D. M Zumbühl, J. D. Zimmerman, and A. C. Gossard, Appl. Phys. Lett. 104, 211106 (2014).

${ }^{31}$ P. Schiffres, IEEE Trans. Electromagn. Compat. 6, 55 (1964).

${ }^{32}$ D. H. Slichter, O. Naaman, and I. Siddiqi, Appl. Phys. Lett. 94, 192508 (2009).

${ }^{33}$ W. Song, M. Rehman, Y. Chong, and S. W. Ryu, J. Korean Phys. Soc. 57, 1490 (2010).

${ }^{34}$ G. Tancredi, S. Schmidlin, and P. J. Meeson, Rev. Sci. Instrum. 85, 026104 (2014).

${ }^{35}$ A. B. Zorin, Rev. Sci. Instrum. 66, 4296 (1995).

${ }^{36}$ H. W. Denny and W. B. Warren, IEEE Trans. Electromagn. Compat. EMC10, 363 (1968)

${ }^{37}$ H. Courtois, O. Buisson, J. Chaussy, and B. Pannetier, Rev. Sci. Instrum. 66, 3465 (1995).

${ }^{38}$ I. Jin, A. Amar, and F. C. Wellstood, Appl. Phys. Lett. 70, 2186 (1997).

${ }^{39}$ H. le Sueur and P. Joyez, Rev. Sci. Instrum. 77, 115102 (2006).

${ }^{40}$ L. Longobardi, D. A. Bennett, V. Patel, W. Chen, and J. E. Lukens, Rev. Sci. Instrum. 84, 014706 (2013).

${ }^{41}$ D. Vion, P. F. Orfila, P. Joyez, D. Esteve, and M. H. Devoret, J. Appl. Phys. 77, 2519 (1995).

${ }^{42}$ C. Debuschewitz, F. Munstermann, V. Kunej, and E. Scheer, J. Low Temp. Phys. 147, 525 (2007).

${ }^{43}$ C. Schirm, H. F. Pernau, and E. Scheer, Rev. Sci. Instrum. 80, 024704 (2009).
${ }^{44}$ R. J. Wenzel, IEEE Trans. Electromagn. Compat. EMC-10, 196 (1968).

${ }^{45}$ P. I. Richards, Proc. Inst. Radio Eng. 36, 217 (1948).

${ }^{46}$ K. Kuroda, J. Inst. Elec. Commun. Engr. (Japan) 36, 10 (1953)

${ }^{47}$ K. Kobayashi, Y. Nemoto, and R. Sato, IEEE Trans. Microwave Theory Tech. 30, 140 (1982)

${ }^{48}$ D. M. Pozar, Microwave Engineering (John Wiley \& Sons, Inc., New York, 2012).

${ }^{49}$ L. Spietz, J. Teufel, and R. J. Schoelkopf, e-print arXiv:cond-mat/0601316 (2006).

${ }^{50}$ See https://www.strem.com/catalog/v/29-0050/17/copper_7440-50-8 for datasheet copper powder CuPA.

${ }^{51}$ See https://www.alfa.com/en/catalog/088390/ for datasheet stainless steel powder SS-316L.

${ }^{52} \mathrm{See}$ https://www.alfa.com/en/elements/Cu/ for datasheet copper powders CuP1-5.

${ }^{53} \mathrm{See} \quad \mathrm{http} / / / \mathrm{www}$. dowa-electronics.co.jp/content/images/function/ferrite powder_130920_en.pdf for datasheets strontium-ferrite powders OP-56, OP-71, UF-S1.

${ }^{54}$ See http://www.ferroxcube.com/FerroxcubeCorporateReception/datasheet/ 3s3.pdf for datasheet MnZn powder 3S3.

${ }^{55}$ See http://shop.tridelta-weichferrite.de/images/MnZn-Ferritpulver.pdf for datasheet MnZn powder TDW.

${ }^{56}$ R. K. Hoffmann, Integrierte Mikrowellenschaltungen (Springer-Verlag, Berlin, Heidelberg, New York, Tokyo, 1983).

${ }^{57}$ J. B. Johnson, Nature 119, 50 (1927).

${ }^{58}$ H. Nyquist, Phys. Rev. 32, 110 (1928).

${ }^{59}$ L. Spietz, K. W. Lehnert, I. Siddiqi, and R. J. Schoelkopf, Science 300, 1929 (2003).

${ }^{60}$ J. Pekola, J. Low Temp. Phys. 135, 723 (2004)

${ }^{61}$ E. Isosaari, T. Holmqvist, M. Meschke, M. Heinonen, and J. P. Pekola, Eur. Phys. J.: Spec. Top. 172, 323 (2009).

${ }^{62}$ N. J. Appleyard, J. T. Nicholls, W. R. Tribe, M. Y. Simmons, and M. Pepper, Phys. E 6, 534 (2000). 